\title{
Indicadores biológicos de suelos lacustres y aluviales de Venezuela bajo diferentes usos. Parte 1. Actividad microbiana y coeficientes ecofisiológicos Biological indicators of lacustrine and alluvial soils in Venezuela under different land use. Part 1. Microbial activity and ecophysiological coefficients
}

\author{
Magaly Ruiz-Dager ${ }^{1:}$ (i) y Jorge Paolini2 $^{\text {(D) }}$ \\ ${ }^{1}$ Centro de Investigación y Extensión en Suelos y Aguas (CIESA). Universidad Nacional Experimental de los Llanos Centrales Rómulo Gallegos (UNERG). Vía \\ El Castrero, San Juan de los Morros. 2301 Estado Guárico, Venezuela. \\ * Autora para correspondencia (magaruizdager@gmail.com) \\ ${ }^{2}$ Centro de Ecología, Instituto Venezolano de Investigaciones Científicas (IVIC). Apartado 21827. 1020-A Caracas, Venezuela.
}

\section{RESUMEN}

El uso del suelo puede afectar la actividad microbiológica y alterar sus transformaciones bioquímicas. En este estudio se examinó la respiración basal y coeficientes ecofisiológicos en suelos venezolanos de la cuenca del lago de Valencia, bajo tres diferentes usos: vegetación natural, monocultivo de caña de azúcar y monocultivo de banano, con el objetivo de determinar la influencia del uso del suelo sobre sus propiedades microbiológicas. El carbono orgánico total $\left(\mathrm{C}_{\text {org }}\right)$, el carbono hidrosoluble y la respiración basal o actividad microbiológica experimentaron una disminución significativa, tanto en suelos aluviales como lacustres, al comparar los suelos agrícolas con los de vegetación nativa. El cociente metabólico $\left(\mathrm{qCO}_{2}\right)$ reflejó diferencias en relación al uso del suelo, siendo mayores los valores en los agroecosistemas bajo banano de acuerdo a la secuencia en sentido decreciente: banano > vegetación natural $>$ caña de azúcar. No obstante, el cociente de eficiencia metabólica $\left(\mathrm{qCO}_{2} / \mathrm{C}_{\text {org }}\right)$ resultó significativamente menor $(P<0.05)$ en los suelos bajo vegetación natural y muestra la interrelación entre el uso más eficiente del carbono y la naturaleza de la materia orgánica aprovechable en dichos suelos. Los resultados revelan que tanto el tipo de cultivo como las prácticas agrícolas ejercen una importante influencia sobre la actividad biológica del suelo.
Palabras clave: banano, caña de azúcar, carbono orgánico, respiración basal.

\section{SUMMARY}

Land use can affect the activities of microorganisms and alter their biochemical transformations. This study examined basal respiration and ecophysiological coefficients in Venezuelan soils in Valencia Lake basin, under three different uses: natural vegetation, sugar cane monoculture and banana monoculture with the aim of determining the influence of land use on soil biological properties. Total organic carbon $\left(\mathrm{C}_{\text {org }}\right)$, water soluble carbon and basal respiration or microbiological activity showed a significant decrease, both in alluvial and lacustrine soils, when agricultural soils were compared with those of native vegetation. The metabolic quotient $\left(\mathrm{qCO}_{2}\right)$ showed differences with relation to land use with higher values in the agroecosystems under banana trees according to the sequence in decreasing order: banana $>$ natural vegetation $>$ sugar cane. Nevertheless, the metabolic efficiency quotient $\left(\mathrm{qCO}_{2} / \mathrm{C}_{\text {org }}\right)$ was significantly lower $(P<0.05)$ in soils under natural vegetation and showed the relationship between the most efficient carbon use and the nature of the available organic matter in these soils. These results reveal that both type of crop and agricultural practices exert an important influence on soil biological activity. 
Index words: banana, sugar cane, organic carbon, basal respiration.

\section{INTRODUCCIÓN}

En las últimas décadas ha crecido el interés a nivel mundial por la sostenibilidad y viabilidad de la agricultura convencional, en la cual la dependencia excesiva del empleo de maquinaria agrícola, y agroquímicos conduce a la destrucción de hábitats naturales, la degradación del suelo, la contaminación del ambiente y a riesgos de salud de las poblaciones. En este sentido la agricultura sostenible apunta a satisfacer los requerimientos de las generaciones actuales y a la vez, garantizar los de las próximas generaciones (NRC, 2010). Las buenas prácticas para el uso y manejo sostenible de los suelos deben permitir rendimientos económicamente y ambientalmente sostenibles, que solo se alcanzarán con la conservación o la restauración de la calidad del medio edáfico (FAO, 2018). Para evaluar la sostenibilidad de un sistema de producción, se deben monitorear las alteraciones que ocurran en los atributos físicos, químicos y biológicos, así como las repercusiones en la capacidad del suelo para servir como medio de sustento para el desarrollo de las plantas y ejercer funciones ambientales (Doran y Safley, 1997).

En numerosas investigaciones se ha encontrado una marcada vinculación entre las propiedades biológicas del suelo y los procesos de degradación y reciclaje de la materia orgánica que ingresa a este medio a través de los restos de origen animal y vegetal. Además, estas propiedades cambian con mayor dinamismo que las propiedades físicas y químicas, por lo que pueden advertir más prontamente sobre los procesos de deterioro o los cambios positivos en la calidad del suelo (Doran y Safley, 1997; Astier-Calderón, Maass y Etchevers, 2002; Cardoso et al., 2013).

La cuenca del lago de Valencia, región centro norte de Venezuela, es una de las áreas más densamente pobladas e industrializadas, donde también se localizan algunos de los mejores suelos agrícolas del país. La cuenca abarca $3.140 \mathrm{~km}^{2}(0.35 \%$ del territorio venezolano) dentro de los estados Aragua y Carabobo, sin embargo, en ella habita el 10\% de la población a nivel nacional, y se mantiene el $30 \%$ de la actividad industrial secundaria. La elevada densidad de población, la alta concentración de industrias y la intensa actividad agrícola, principalmente como monocultivo en la región, son las principales causas de los problemas ambientales en la zona, cuya consecuencia directa es una acelerada eutrofización del lago. Aun cuando los suelos de la cuenca son muy fértiles y productivos, el manejo agrícola inapropiado podría conducir a su degradación y permanente deterioro. La sostenibilidad de estos suelos es importante para preservar en ellos la producción de alimentos y fibras y, por lo tanto, el estudio de indicadores sensibles de la degradación del suelo resulta de capital relevancia; de allí que se haya planteado la evaluación de los atributos microbiológicos, los cuales han sido considerados como indicadores sensibles de las alteraciones ocasionadas por el cultivo y las prácticas asociadas a él, así como por la contaminación.

El objetivo de este trabajo fue determinar la respiración basal y sus coeficientes ecofisiológicos en suelos bajo vegetación nativa y monocultivos de caña de azúcar y banano, ubicados en la cuenca del lago de Valencia (Venezuela), a fin de evaluar la influencia del uso del suelo sobre sus atributos biológicos.

\section{MATERIALES Y MÉTODOS}

Los sitios donde se tomaron las muestras están localizados en las áreas llanas de la cuenca del lago de Valencia, la cual está delimitada por los meridianos de longitud oeste $67^{\circ} 10^{\prime}-68^{\circ} 10^{\prime}$, y por los paralelos de latitud norte $10^{\circ} 00^{\prime}-10^{\circ} 20^{\prime}$. La clasificación climática de este sitio corresponde a tropical húmedo. En esta zona se presenta una distribución unimodal de la precipitación, con dos períodos claramente marcados, uno seco caracterizado por bajas precipitaciones (enero, febrero y marzo), y otro húmedo o de altas precipitaciones (junio, julio, y agosto), y la media anual varía entre $900 \mathrm{~mm}$ y $1150 \mathrm{~mm}$. El promedio de temperatura anual es $24.6{ }^{\circ} \mathrm{C}$ (Guevara, Guevara y García, 2008).

Para este estudio se seleccionaron suelos lacustres y aluviales (Cuadro 1 y Figura 1). Los primeros se han clasificado como MOLLIC USTIFLUVENTS, francosa, carbonática, mixta, isohipertérmica, mientras que los suelos aluviales entran en la clasificación de FLUVENTIC USTROPEPTS, francosa gruesa mixta isohipertérmica, con la salvedad del suelo AB2 que corresponde a un FLUVENTIC HAPLUSTOLLS (Elizalde, Viloria y Rosales, 2007; Ruiz y Paolini, 2004) (Cuadro 1). 
Cuadro 1. Características de los suelos estudiados ${ }^{\dagger}$, en la cuenca del lago de Valencia (Venezuela).

Table 1. Characteristics of the studied lacustrine and alluvial soils ${ }^{\dagger}$, in Valencia Lake basin (Venezuela).

\begin{tabular}{|c|c|c|c|c|c|c|}
\hline $\begin{array}{l}\text { Origen del } \\
\text { suelo }\end{array}$ & Uso del suelo & Siglas & Sector (Finca) & Tipo de agua de riego & $\mathrm{pH}$ & $\begin{array}{l}\text { C-Biomasa } \\
\text { microbiana }\end{array}$ \\
\hline & & & & & $01: 2.5$ & $\mathrm{mg} \mathrm{Cmic} \mathrm{kg}{ }^{-1}$ \\
\hline \multirow{5}{*}{ Lacustre } & \multirow[t]{2}{*}{ Vegetación nativa } & LVN & $\begin{array}{l}\text { PAVECA } \\
\text { (Torumo) }\end{array}$ & Ninguna & 6.97 & 1552.7 \\
\hline & & $\mathrm{LC} 1$ & $\begin{array}{l}\text { Las Palmeras } \\
\text { (El Cedro) }\end{array}$ & $\begin{array}{l}\text { Lago de Valencia } \\
\text { (no tratada) }\end{array}$ & 7.68 & 630.9 \\
\hline & \multirow[t]{2}{*}{ Caña de azúcar } & $\mathrm{LC} 2$ & $\begin{array}{l}\text { Las Palmeras } \\
\text { (El Cedro) }\end{array}$ & Pozo profundo & 7.64 & 623.1 \\
\hline & & LC3 & $\begin{array}{l}\text { Las Palmeras } \\
\text { (El Cedro) }\end{array}$ & Residual no tratada & 7.41 & 1356.2 \\
\hline & Banano & LB1 & Caño Rico & $\begin{array}{l}\text { Embalse Taigüaigüay } \\
\text { (tratada) }\end{array}$ & 7.91 & 755.6 \\
\hline \multirow{5}{*}{ Aluvial } & Vegetación nativa & AVN & $\begin{array}{l}\text { Santa Cruz de Aragua } \\
\text { (La Soledad) }\end{array}$ & Ninguna & 6.96 & 1031.2 \\
\hline & \multirow{2}{*}{ Caña de azúcar } & $\mathrm{AC} 1$ & $\begin{array}{l}\text { Santa Cruz de Aragua } \\
\text { (Los Aguirre) }\end{array}$ & $\begin{array}{l}\text { Río Aragua } \\
\text { (residual no tratada) }\end{array}$ & 7.54 & 634.2 \\
\hline & & $\mathrm{AC} 2$ & $\begin{array}{l}\text { Santa Cruz de Aragua } \\
\text { (La Josefa) }\end{array}$ & $\begin{array}{l}\text { Embalse Zuata } \\
\text { (tratada) }\end{array}$ & 7.52 & 700.8 \\
\hline & \multirow{2}{*}{ Banano } & $\mathrm{AB} 1$ & $\begin{array}{l}\text { Santa Cruz de Aragua } \\
\text { (La Soledad) }\end{array}$ & $\begin{array}{l}\text { Embalse Zuata } \\
\text { (tratada) }\end{array}$ & 7.59 & 205.8 \\
\hline & & AB2 & $\begin{array}{l}\text { Palo Negro } \\
\text { (Morales) }\end{array}$ & $\begin{array}{l}\text { Río Turmero } \\
\text { (residual no tratada) }\end{array}$ & 7.78 & 244.8 \\
\hline
\end{tabular}

${ }^{\dagger}$ Cuadro elaborada a partir de los datos reportados por Ruiz y Paolini (2004). ${ }^{\ddagger}$ Determinado por el método de respiración inducida por sustrato.

$\dagger$ Table made from the data reported by Ruiz and Paolini (2004). ${ }^{\star}$ Determined by the substrate-induced respiration method.

En cada uno de los dos tipos de suelos se ubicaron tres usos de la tierra diferentes: vegetación natural o nativa, monocultivo de caña de azúcar y monocultivo de banano. En los suelos con vegetación nativa predominan especies arbóreas y arbustos autóctonos de la zona. Esos suelos nunca habían sido objeto de ninguna práctica agrícola, por tanto, se estudiaron como suelos testigos o control. En los suelos de origen lacustre, la caña de azúcar y el banano fueron cultivados por un período de 13 y 20 años respectivamente; mientras que, en los suelos aluviales, la caña de azúcar se había establecido desde hacía cuarenta y tres años y el banano tenía seis años sembrado (previamente se producía maíz en estos últimos, por un período mayor de una década). En los suelos agrícolas prevalecía un manejo convencional, con uso de fertilizantes químicos y pesticidas y en la mayoría de los casos se empleaban para irrigación, aguas residuales sin tratamiento o solo con tratamiento primario (Cuadro 1). Adicionalmente, los suelos bajo banano recibían gallinaza como abono orgánico. En el manejo de la caña de azúcar siempre se practicó la quema antes de la cosecha (Ruiz y Paolini, 2004).

Las muestras de suelo se tomaron a una profundidad de $0-5 \mathrm{~cm}$. Para cada tipo de uso de la tierra se colectaron submuestras de igual volumen en 20 puntos diferentes del área a considerar en un patrón en zig-zag y las mismas se mezclaron para obtener tres muestras compuestas. Las muestras compuestas se dividieron en dos porciones. Una de las porciones se secó al aire, se desmenuzó y se tamizó a $2 \mathrm{~mm}$ para el análisis fisicoquímico ( $\mathrm{pH}, \mathrm{C}_{\text {org }}$ y CHS). La otra porción se conservó con la humedad de campo, se tamizó a $2 \mathrm{~mm}$ y se guardó en bolsas de polietileno bajo refrigeración a $4{ }^{\circ} \mathrm{C}$ para su posterior análisis de la respiración basal o actividad microbiana en un lapso no mayor de dos semanas (Ruiz y Paolini, 2004). 


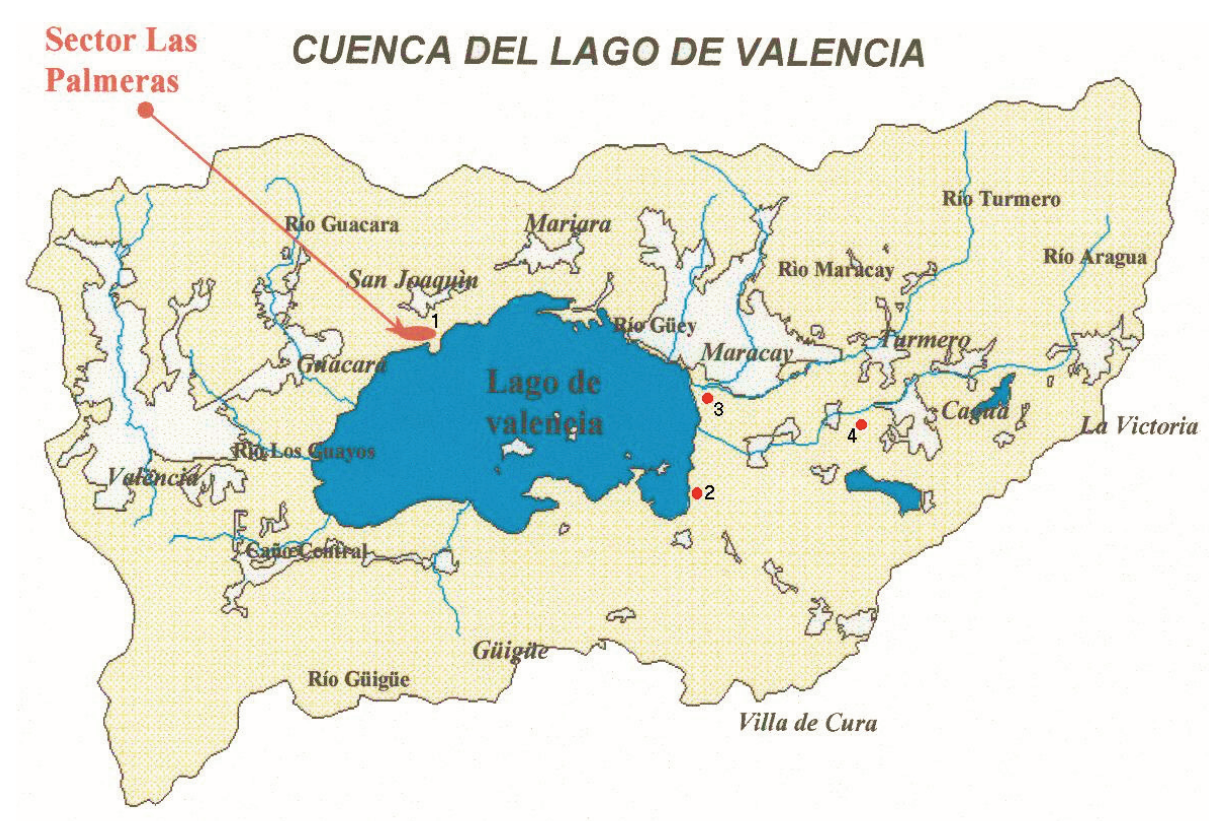

Figura 1. Zonas de la cuenca del lago de Valencia (Venezuela) en las que se encuentran los suelos seleccionados para este estudio. 1: Guacara; 2: Caño Rico; 3: Palo Negro; 4: Santa Cruz.

Figure 1. Areas of Lake Valencia (Venezuela) where the soils selected for this study are located. 1: Guacara; 2: Caño Rico; 3: Palo Negro; 4: Santa Cruz.

El carbono orgánico total $\left(\mathrm{C}_{\text {org }}\right)$ se determinó por la oxidación húmeda de la muestra de suelo con una mezcla de dicromato de potasio y ácido sulfúrico sin aplicación de calor externo. En la reacción de oxidación el $\mathrm{Cr}(+6)$ es reducido a $\mathrm{Cr}(+3)$ de color verde, cuya concentración se mide por espectrofotometría a $\lambda=$ 600 nm (Heanes, 1984; Paolini, 2018). Para extraer el carbono hidrosoluble (CHS) se agitaron muestras secas en tubos de centrifuga, con una relación suelo/ agua de 1:10 durante 1 hora. Después de centrifugarlas por 15 minutos, a 3500 revoluciones por minuto, se procedió a pasar el sobrenadante por un filtro de membrana marca Millipore de $0.45 \mathrm{~m} \mu$. Posteriormente se determinó el carbono orgánico hidrosoluble por combustión con oxidación catalítica en un analizador de carbono marca Shimadzu 5000A.

Para la cuantificación de la respiración basal se siguió el procedimiento descrito por Paolini (2018), según el cual, una muestra de suelo se ajusta al $60 \%$ de su capacidad de retención de humedad, se incuba en oscuridad a temperatura ambiente de laboratorio $\left(22{ }^{\circ} \mathrm{C}\right)$ en un recipiente hermético que contiene una trampa de álcali y posteriormente el $\mathrm{CO}_{2}$ liberado durante dos días, se precipita con $\mathrm{BaCl}_{2}$ y la cantidad de solución alcalina que no reacciona es titulada con un ácido valorado, usando fenolftaleína como indicador. Con los parámetros determinados anteriormente se calcularon los siguientes coeficientes ecofisiológicos: cociente metabólico $\left(\mathrm{qCO}_{2}=\mathrm{RB} / \mathrm{C}_{\text {mic }}\right)$ y coeficiente de eficiencia metabólica $\left(\mathrm{qCO}_{2} / \mathrm{C}_{\text {org }}\right)$.

Todos los análisis fueron realizados por triplicado y se empleó el programa STATISTIX para Windows versión 8.0 (2003) en el procesamiento estadístico de los datos. Para estimar los supuestos de normalidad se utilizó la prueba de Wilk-Shapiro. Las variaciones de los parámetros microbiológicos de acuerdo al uso del suelo (vegetación natural, cultivo de caña de azúcar, cultivo de banano), se evaluaron mediante la aplicación de la prueba no paramétrica de Kruskall-Wallis, debido a que los datos no seguían una distribución normal. Todos los supuestos y pruebas se validaron con un nivel de significancia del 95\%. Los resultados se expresaron con respecto al peso de suelo seco.

\section{RESULTADOS Y DISCUSIÓN}

Los valores obtenidos para el $\mathrm{C}_{\text {org }}$, CHS y las propiedades microbiológicas de los suelos estudiados se muestran en el Cuadro 2. En un trabajo previo fueron discutidos los valores del carbono de la biomasa 
Cuadro 2. Valores promedios de las propiedades químicas $\left(\mathrm{C}_{\text {org }}\right.$ y CHS), respiración basal (RB) y coeficientes metabólicos de los suelos estudiados $(n=3)$.

Table 2. Mean values of chemical properties ( $\mathrm{C}_{\text {org }}$ and $\left.\mathrm{CHS}\right)$, basal respiration (RB) and metabolic coefficients of the studied soils $(\mathbf{n}=3)$.

\begin{tabular}{|c|c|c|c|c|c|}
\hline Suelo & $\mathrm{C}_{\text {org }}$ & CHS & $\mathrm{RB}$ & $\mathrm{qCO}_{2}$ & $\mathrm{qCO}_{2} /$ Corg \\
\hline & $\mathrm{g} \mathrm{kg}^{-1}$ & $\mathrm{mg} \mathrm{kg}^{-1}$ & $\mu \mathrm{g} \mathrm{C}-\mathrm{CO}_{2} \mathrm{~g}^{-1} \mathrm{~h}^{-1}$ & $\mathrm{mg} \mathrm{C}-\mathrm{CO}_{2} \mathrm{~g}^{-1} \mathrm{C}_{\text {mic }} \mathrm{h}^{-1}$ & mg C-CO $\mathrm{g}^{-1} \mathrm{C}_{\text {mic }} \mathrm{h}^{-1}\left(\mathrm{~g} \mathrm{C} \mathrm{g}^{-1} \text { suelo }\right)^{-1}$ \\
\hline LVN & 81.4 & 941 & 6.61 & 4.3 & 52.2 \\
\hline LC1 & 17.2 & 362 & 1.37 & 2.2 & 128.8 \\
\hline LC2 & 17.1 & 285 & 1.80 & 2.9 & 171.9 \\
\hline LC3 & 32.9 & 375 & 4.36 & 3.2 & 97.7 \\
\hline LB1 & 54.0 & 459 & 3.40 & 4.5 & 83.5 \\
\hline AVN & 47.5 & 893 & 2.58 & 2.5 & 54.7 \\
\hline $\mathrm{AC} 1$ & 19.9 & 272 & 1.24 & 2.0 & 98.8 \\
\hline $\mathrm{AC} 2$ & 21.1 & 278 & 1.27 & 1.8 & 89.4 \\
\hline AB1 & 8.9 & 257 & 0.75 & 3.6 & 408.9 \\
\hline AB2 & 12.9 & 256 & 1.17 & 4.8 & 370.1 \\
\hline
\end{tabular}

$\mathrm{C}_{\text {org }}=$ carbono orgánico total; $\mathrm{CHS}=$ carbono hidrosoluble; $\mathrm{RB}=$ respiración basal; $\mathrm{qCO}_{2}=$ cociente metabólico; $\mathrm{qCO}_{2} / \mathrm{C}_{\text {org }}=$ coeficiente de eficiencia metabólica; $\mathrm{C}_{\mathrm{mic}}=$ carbono de la biomasa microbiana.

$\mathrm{C}_{\text {org }}^{\text {mic }}=$ total organic carbon; $\mathrm{CHS}$ = water-soluble carbon; $\mathrm{RB}=$ basal breathing; $\mathrm{qCO}_{2}=$ metabolic ratio; $\mathrm{qCO}_{2} / \mathrm{C}_{\text {org }}=$ metabolic efficiency coefficient; $\mathrm{C}_{\text {mic }}=$ carbon from microbial biomass.

microbiana $\left(\mathrm{C}_{\text {mic }}\right)$ y el cociente microbiano $\left(\mathrm{C}_{\text {mic }} / \mathrm{C}_{\text {org }}\right)$ (Ruiz y Paolini, 2004). De acuerdo a los resultados de esa investigación, el carbono de la biomasa microbiana $\left(\mathrm{C}_{\text {mic }}\right)$ mostró diferencias significativas $(P<0.05)$ entre los 3 usos del suelo, en el orden decreciente: vegetación natural $>$ caña de azúcar $>$ banano. Con respecto al suelo bajo vegetación nativa, el $\mathrm{C}_{\text {mic }}$ experimentó una disminución promedio del orden de $37 \%$ en el caso del suelo bajo caña de azúcar y de $68 \%$ en el suelo sembrado con banano. El cociente microbiano $\left(\mathrm{C}_{\text {mic }} /\right.$ $\mathrm{C}_{\text {org }}$ ) no fue estadísticamente diferente entre los suelos con vegetación nativa y los suelos bajo banano, los cuales exhibieron valores más bajos que los de los suelos sembrado con caña de azúcar.

En el Cuadro 3 se presentan las medias de las concentraciones de $\mathrm{C}_{\text {org }}$ y CHS, de la $\mathrm{RB}$ y los coeficientes metabólicos, agrupados de acuerdo al uso del suelo (vegetación natural, cultivo de caña de azúcar, cultivo de banano).

La concentración de $\mathrm{C}_{\text {org }}$ promedio en los suelos control es 2.98 veces mayor que el de los suelos cultivados con caña de azúcar y 2.6 veces mayor que los que se encontraban sembrados con banano, lo que equivale a una disminución de 66.5 y $60.7 \%$ en el $\mathrm{C}_{\text {org }}$ cuando se compara el suelo bajo vegetación nativa con los suelos cultivados (Cuadro 3). Hallazgos similares se encuentran en la literatura para suelos de las regiones tropicales cultivados con bananos y caña de azúcar (Borges et al., 2014; Cherubin et al., 2016; Dominy, Haynes y van Antwerpen, 2002; Evangelista, Partelli, Ferreira y Pires, 2013; Fialho, Gomes, Oliveira y Silva Júnior, 2006; Novak, Carvalho, Santiago y Portilho, 2017; Paredes, Portilho y Mercante, 2015; Sant'Anna, Fernandes, Ivo y Costa, 2009; Souza et al., 2012). Por otro lado, se ha señalado que los tenores de materia orgánica de suelos tropicales sometidos a continuo cultivo disminuyen entre 30 y $60 \%$ al compararlos con los de los sitios en que se ha conservado la vegetación nativa, en el transcurso de unos pocos años. Tal como lo expresa Casanova (2005), cuando en un suelo se elimina su vegetación natural para sembrar cultivos agrícolas, ocurre una disminución de su contenido de materia orgánica debido en gran medida a que la mayor parte del cultivo es cosechado con fines de alimentación humana y animal siendo poco los restos vegetales que retornan al suelo, mientras que en el suelo virgen casi toda la materia orgánica producida por la vegetación natural regresa al suelo vía hojarasca y 
Cuadro 3. Valores promedios de las propiedades químicas $\left(\mathrm{C}_{\text {org }}\right.$ y CHS), respiración basal (RB) y sus coeficientes metabólicos de los suelos estudiados agrupando la información según el uso del suelo.

Table 3. Mean values of the chemical properties $\left(\mathrm{C}_{\text {org }}\right.$ and CHS), basal respiration (RB) and their metabolic coefficients of the studied soils grouped according to soil use.

\begin{tabular}{|c|c|c|c|}
\hline \multirow[b]{2}{*}{ Variable } & \multicolumn{3}{|c|}{ Uso del suelo } \\
\hline & $\begin{array}{l}\text { Vegetación natural } \\
\qquad(\mathrm{n}=6)\end{array}$ & $\begin{array}{l}\text { Caña de azúcar } \\
\quad(\mathrm{n}=15)\end{array}$ & $\begin{array}{l}\text { Banano } \\
(\mathrm{n}=9)\end{array}$ \\
\hline Carbono orgánico total $\left(\mathrm{C}_{\text {org }}\right) \mathrm{g} \mathrm{kg}^{-1}$ & $64.4 \pm 24.0 \mathrm{a}$ & $21.6 \pm 2.0 \mathrm{~b}$ & $25.3 \pm 25.0 \mathrm{~b}$ \\
\hline Carbono hidrosoluble ( $\mathrm{mg} \mathrm{C} \mathrm{kg}^{-1}$ ) & $917 \pm 34 \mathrm{a}$ & $314 \pm 42 b$ & $324 \pm 117 b$ \\
\hline Respiración basal $\left(\mu \mathrm{g} \mathrm{C}-\mathrm{CO}_{2} \mathrm{~g}^{-1} \mathrm{~h}^{-1}\right)$ & $4.6 \pm 2.9 \mathrm{a}$ & $2.0 \pm 0.3 b$ & $1.8 \pm 1.4 \mathrm{~b}$ \\
\hline $\mathrm{qCO}_{2}\left(\mathrm{mgC}-\mathrm{CO}_{2} \mathrm{~g}^{-1} \mathrm{C}_{\text {mic }} \mathrm{h}^{-1}\right)$ & $3.4 \pm 1.2 \mathrm{~b}$ & $2.4 \pm 0.5 \mathrm{c}$ & $4.3 \pm 0.6 \mathrm{a}$ \\
\hline 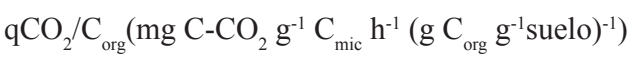 & $53 \pm 0.3 \mathrm{~b}$ & $117 \pm 37 \mathrm{a}$ & $287 \pm 178$ a \\
\hline
\end{tabular}

En cada fila, medias seguidas por la misma letra no son estadísticamente diferentes a un nivel de significación del 5\%, según la prueba de Kruskal Wallis. In each row, means followed by the same letter are not statistically different at a significance level of 5\%, according to Kruskal Wallis' test.

exudados radiculares. Además, en los suelos cultivados se incrementa la erosión, y las prácticas de labranza intensiva degradan los agregados de suelo, con lo cual queda expuesto el carbono orgánico asociado a ellos, a su degradación por parte de los microorganismos (Franzluebbers, 2004). También otros factores que condicionan las transformaciones de la materia orgánica mediadas por los microorganismos pueden ser modificados por la conversión de vegetación nativa a cultivos agrícolas como lo son el potencial redox, la retención de humedad y la temperatura (FAO, 2017). La magnitud de estos cambios depende tanto del nivel previo de materia orgánica como del sistema de manejo agrícola. En suelos venezolanos, HernándezHernández et al. (2013) observaron que la materia orgánica se redujo entre 20 y $80 \%$ al transformar el bosque natural en monocultivos de cítricos, pinos o eucaliptus y entre 16 y $20 \%$ por la transformación de sabana nativa a pastizal. En México, se encontraron pérdidas del $18 \%$ en la concentración del $\mathrm{C}_{\text {org }}$ del suelo cuando los bosques fueron convertidos en pastizales (García-Oliva, Gallardo, Montaño y Islas, 2006). En este estudio, el análisis de varianza no reflejó diferencias significativas $(P<0.05)$ en cuanto a las concentraciones de $\mathrm{C}_{\text {org }}$ entre los suelos bajo cultivo de banano y los suelos sembrados con caña de azúcar (Cuadro 3). Es posible que esto se deba al hecho de que los suelos cultivados estudiados guardan similitud en el período que tienen sembrados, aunque con rubros diferentes. En algunos de los casos, se produjeron con anterioridad rubros distintos a los existían para el momento en que se realizó esta investigación, por ejemplo, en los suelos aluviales el banano tenía seis años sembrado, pero previamente se producía maíz en ellos. Otro hecho es que en ninguno de los manejos se contempló la práctica de incorporación de residuos de cosecha, sino que, al contrario, los mismos eran removidos del área. Tampoco se consideró la adición de otra enmienda orgánica a excepción de la gallinaza en los suelos bajo banano, en los que se consideraba como un complemento a la fertilización química, a la que siempre se concedió prioridad. Además, el clima de la zona es el mismo para ambos usos del suelo y los valores de $\mathrm{pH}$ encontrados resultaron muy similares. Es conocido que estos dos últimos factores influyen en la concentración del carbono orgánico por ser determinantes en la biomasa microbiana y su actividad.

El carbono hidrosoluble (CHS) mostró la misma tendencia que el $\mathrm{C}_{\text {org }}$ al presentar valores significativamente mayores $(P<0.05)$ en los sitios de vegetación nativa con relación a las áreas cultivadas y no registrar diferencias estadísticamente significativas entre los suelos bajo banano o caña de azúcar. Para los tres usos, la proporción del CHS respecto al $\mathrm{C}_{\text {org }}$ fue similar, alrededor de $1.5 \%$. El CHS comprende compuestos muy lábiles, de bajo peso molecular, entre los que se encuentran aminoácidos, lípidos y azúcares, que son fácilmente aprovechables para los microorganismos e incentivan su crecimiento y sus funciones. Se ha considerado que esta fracción aumenta 
con la incorporación de materia orgánica a través de abonos orgánicos y de hojarasca (Paolini, 2018). Los datos de este estudio para suelos cultivados están dentro del ámbito reportado para suelos tropicales, así Mazzarino, Szott y Jimenez (1993) en Costa Rica para suelos bajo rotación de maíz-frijol señalan tenores entre 205 y $284 \mathrm{mg} \mathrm{C} \mathrm{kg}^{-1}$; Rivera-Cruz et al. (2008) en México para suelos bajo banano inoculados con un consorcio de microorganismos reportan valores comprendidos entre 182 y 309 mg C kg-1; Alguacil, Lozano, Campoy y Roldán (2010) encontró valores comprendidos entre 92 y $124 \mathrm{mg} \mathrm{C} \mathrm{kg}^{-1}$ para suelos de sabanas en los Llanos Centrales de Venezuela y recientemente por Paolini (2018) en cafetales de Venezuela bajo manejo tradicional $\left(351 \pm 111 \mathrm{mg} \mathrm{C} \mathrm{kg}^{-1}\right)$ y convencional $\left(177 \pm 52 \mathrm{mg} \mathrm{C} \mathrm{kg}^{-1}\right)$, aunque para cafetales bajo manejo orgánico resultaron ser mayores $(872 \pm$ $134 \mathrm{mg} \mathrm{C} \mathrm{kg}^{-1}$ ). En un estudio realizado en Australia, en el que se compararon los niveles de $\mathrm{C}_{\text {org }}$ y CHS en suelos bajo dos prácticas de manejo de caña de azúcar, convencional y nuevo (que incluye rotación de cultivo, mínima labranza, retención de residuos y ausencia de quema pre-cosecha) y en suelos sembrados con pasto, se encontró que los contenidos de CHS variaron en la secuencia: pasto $\left(211 \mathrm{mg} \mathrm{C} \mathrm{kg}^{-1}\right)>$ caña de azúcar manejo nuevo (130 $\left.\mathrm{mg} \mathrm{C} \mathrm{kg}^{-1}\right) \geq$ caña de azúcar manejo convencional (117 $\mathrm{mg} \mathrm{C} \mathrm{kg}^{-1}$ ), aunque no se observaron diferencias significativas entre los dos manejos de caña de azúcar (Stirling, Moody y Stirling, 2010), estos valores resultaron ser inferiores a los de este estudio. En relación al cultivo de banano en suelos tropicales (0-30 cm) de China, Zhong, Zeng y Jin (2015), obtuvieron niveles de CHS significativamente más altos bajo rotaciones banano-piña $\left(737 \mathrm{mg} \mathrm{C} \mathrm{kg}^{-1}\right) \mathrm{y}$ banano-papaya (663 $\mathrm{mg} \mathrm{C} \mathrm{kg}^{-1}$ ) que en el monocultivo de banano (480 $\mathrm{mg} \mathrm{C} \mathrm{kg}^{-1}$ ), valores que superan a los de este estudio.

La respiración basal o la actividad microbiológica se ha empleado frecuentemente como un bioindicador de la salud del suelo en ecosistemas forestales y agrícolas. Todos los microorganismos heterótrofos participan en el proceso de degradación de los compuestos orgánicos del suelo. La tasa a la cual ocurre ese proceso se ha considerado como un indicador del estado biológico de este medio (Nannipieri, Greco y Ceccanti, 1990).

Los cambios en la vegetación como la deforestación reducen la respiración microbiana a largo plazo debido al decrecimiento de las entradas de carbono orgánico en el suelo a través de la hojarasca o la rizosfera (Bini et al., 2013). La respiración basal (RB) presentó valores significativamente superiores $(P<0.05)$ en los suelos bajo vegetación nativa, mientras que entre los suelos cultivados con caña de azúcar y banano no se evidenciaron diferencias (Cuadro 3). Esto podría estar relacionado con la entrada de materiales orgánicos más abundante y de naturaleza heterogénea en los suelos bajo vegetación natural, lo cual se traduce en una mayor actividad de la microbiota heterótrofa.

Los valores de este estudio para la respiración basal son similares a los señalados en suelos tropicales cultivados con caña de azúcar y bananos (Acuña et al., 2006; Da Silva et al., 2012; Fialho et al., 2006; Novak et al., 2017; Pôrto, Alves, Diniz, Souza y Santos, 2009; González-García, González, Atencio y Soto, 2021), aunque superan en caso de la caña azúcar bajo quema y mecanización en Brasil (Evangelista et al., 2013; Paredes et al., 2015) o en zonas salinizadas de Sudáfrica (Rietz y Haynes, 2003).

El cociente metabólico $\left(\mathrm{qCO}_{2}\right)$ se interpreta como la cantidad de $\mathrm{C}-\mathrm{CO}_{2}$ respirado por unidad de carbono de la biomasa microbiana en una unidad de tiempo, lo que significa que la actividad de los microorganismos es más eficiente en la medida en que se pierda menos carbono a través de la respiración (Anderson, 2003; Dilly, 2005). Valores más altos de $\mathrm{qCO}_{2}$ indican que las comunidades microbianas están sometidas a condiciones adversas, en las que los microorganismos gastan más energía (es decir, compuestos de carbono) para su mantenimiento en detrimento del crecimiento de la población (Anderson y Domsch, 2010). El $\mathrm{qCO}_{2}$ reflejó diferencias $(P<0.05)$ en relación al uso del suelo, siendo mayores los valores en los agroecosistemas bajo banano de acuerdo a la secuencia en sentido decreciente: banano $>$ vegetación natural $>$ caña de azúcar. Es posible que en ello haya influido el hecho de que los suelos sembrados con banano reciben gallinaza periódicamente, lo cual coincidiría con los hallazgos de Goyal, Mishra, Dhankar, Kapoor y Batra (1993), quienes encuentran valores más altos de $\mathrm{qCO}_{2}$ en suelos agrícolas de India a los que se ha adicionado estiércol animal. Se ha señalado que la adición de residuos orgánicos frescos coloca a la población microbiana bajo estrés, y ésta, para sobrevivir al ambiente hostil, desarrolla mecanismos de defensa incrementando su respiración por unidad de biomasa (Anderson y Domsch, 1993; Dilly, 2005). El uso de plaguicidas en mayor proporción en el cultivo de banano también podría contribuir al valor más alto del $\mathrm{qCO}_{2}$ en esos 
suelos, tal como se ha evidenciado en algunos estudios, en los que se han encontrado los mayores valores de $\mathrm{qCO}_{2}$ en aquellos suelos donde se emplean altas dosis de agroquímicos (Silva et al., 2010). En todo caso, los valores más elevados del $\mathrm{qCO}_{2}$ en los suelos sembrados con banano indican una menor eficiencia de la microbiota al utilizar el carbono orgánico en ese agroecosistema. El resultado del $\mathrm{qCO}_{2}$ obtenido para los suelos control (mayor que el de los suelos bajo caña de azúcar), concuerda con los hallazgos de Saviozzi, Levi, Cardelli y Riffaldi (2001) y Francaviglia, Renzi, Ledda y Benedetti (2017) en Italia, quienes señalan valores de $\mathrm{qCO}_{2}$ más altos en suelos bajo vegetación nativa que en suelos cultivados. El suelo control lacustrino $(\mathrm{LVN})$ presenta un valor elevado de $\mathrm{qCO}_{2}$ (Cuadro 2), es posible que este hecho se encuentre relacionado con su alto contenido natural de sales, lo cual se refleja en el mayor valor de la conductividad eléctrica $\left(12.2 \mathrm{dS} \mathrm{m}^{-1}\right)$ para todos los suelos bajo estudio. Esa particularidad del suelo LVN puede significar una situación de estrés para la población microbiana, y ello se estaría evidenciando a través del alto valor del $\mathrm{qCO}_{2}$. Este caso proporciona evidencia de que el $\mathrm{qCO}_{2}$ es un índice problemático porque responde de manera impredecible a cualquier perturbación (Saviozzi et al., 2001; Wardle y Ghani, 1995).

El cociente de eficiencia metabólica $\left(\mathrm{qCO}_{2} /\right.$ $\mathrm{C}_{\text {org }}$ ) es un índice propuesto por Dilly, Winter, Lang y Munch (2001) y Dilly (2005) que representa el cociente metabólico normalizado por el contenido de carbono orgánico total. Este parámetro tiene la ventaja de integrar en un solo valor, la respiración basal, el carbono de la biomasa microbiana y el contenido de carbono orgánico total. Su significado implica que mientras menor es su magnitud, mayor será la eficiencia de la población microbiana desde el punto de vista energético. El valor de $\left(\mathrm{qCO}_{2} / \mathrm{C}_{\text {org }}\right)$ aumenta bien sea, en la medida en que la respiración se incrementa, decrezca la biomasa microbiana o disminuya la cantidad o calidad del carbono orgánico del sustrato (Dilly, 2005). En el presente estudio el cociente $\mathrm{qCO}_{2} / \mathrm{C}_{\text {org }}$ resultó significativamente menor $(P<0.05)$ en los sitios con vegetación nativa en comparación con las áreas con monocultivo, lo que revela un uso más eficiente del carbono por parte de la microbiota en los suelos control. Los valores promedio de este cociente en los tres usos de suelo evaluados se encuentran entre 53 y $287 \mathrm{mg} \mathrm{C}-\mathrm{CO}_{2} \mathrm{~g}^{-1} \mathrm{C}_{\text {mic }} \mathrm{h}^{-1}$ (g C g ${ }^{-1}$ suelo) $)^{-1}$ (Cuadro 3). Estos datos concuerdan con los indicados por Dilly et al. (2001) para suelos agrícolas y forestales de Alemania, en el intervalo comprendido entre 20 y $274 \mathrm{mg} \mathrm{C}-\mathrm{CO}_{2} \mathrm{~g}^{-1} \mathrm{C}_{\text {mic }} \mathrm{h}^{-1}$ ( $\mathrm{g} \mathrm{C} \mathrm{g}^{-1}$ suelo) ${ }^{-1}$. De acuerdo a estos autores, cuando el medio es adverso, los microorganismos tendrán mayores requerimientos energéticos para mantener su biomasa y entonces, los valores del $\mathrm{qCO}_{2}$ aumentan y se pierde carbono. Igualmente señalan que el valor del $\mathrm{qCO}_{2}$ se incrementa al aumentar la fracción lábil de la materia orgánica $\mathrm{y}$, por tanto, el cociente $\mathrm{qCO}_{2} / \mathrm{C}_{\text {org }}$ hace referencia a la interrelación entre la eficiencia en el uso del carbono y la calidad de la materia orgánica disponible en el suelo. Ese estudio llevado a cabo en suelos de Alemania, mostró diferencias significativas en la eficiencia del uso del carbono entre los sitios estudiados, principalmente debido al clima, la textura y el uso del suelo, por lo que los autores propusieron el cociente $\mathrm{qCO}_{2} / \mathrm{C}_{\text {org }}$ como un indicador capaz de detectar las alteraciones asociadas con el uso del suelo. Este índice fue aplicado recientemente por Paolini (2018) en Venezuela, al comparar diversas formas de manejo del café; donde el café cultivado a plena exposición solar y aplicación de fertilizantes (manejo convencional) presentó los mayores valores de este índice en comparación con suelos bajo manejo orgánico (certificación orgánica) y manejo tradicional.

\section{CONCLUSIONES}

Este estudio demuestra que la conversión del bosque nativo a monocultivos (caña de azúcar y bananos) afecta de forma negativa las propiedades químicas y microbiológicas de los suelos en la cuenca del lago de Valencia. En este sentido, el carbono orgánico total, el carbono hidrosoluble y la respiración basal o actividad microbiológica experimentaron una disminución significativa, tanto en suelos aluviales como lacustres, al comparar los suelos cultivados con los de vegetación natural. El cociente metabólico $\left(\mathrm{qCO}_{2}\right)$ reflejó diferencias en relación al uso del suelo, siendo mayores los valores en los agroecosistemas bajo banano de acuerdo a la secuencia en sentido decreciente: banano $>$ vegetación natural $>$ caña de azúcar. Sin embargo, el cociente de eficiencia metabólico $\left(\mathrm{qCO}_{2} / \mathrm{C}_{\text {org }}\right)$ resultó significativamente menor $(P<0.05)$ en las zonas de bosque nativo que, en los sitios con monocultivos, lo que revela un uso más eficiente del carbono por parte de la microbiota en los suelos bajo vegetación nativa. Los resultados del presente estudio mostraron 
que las propiedades microbiológicas y los parámetros metabólicos derivados son indicadores sensibles para estimar la calidad de los suelos y dado su bajo costo y facilidad de medición podrían ser usados para evaluar de forma rápida cambios en nuevas prácticas agrícolas en la zona estudiada como por ejemplo reducción de fertilizantes inorgánicos, uso de enmiendas orgánicas estables y maduras y biofertilizantes, uso de sistemas de riego en ambos cultivos; supresión del fuego en la cosecha de caña de azúcar entre otras para así aumentar la productividad y tener un manejo más cónsono con el ambiente.

\section{DECLARACIÓN DE ÉTICA}

No aplicable.

\section{CONSENTIMIENTO PARA PUBLICACIÓN}

No aplicable.

\section{DISPONIBILIDAD DE DATOS}

El conjunto de datos utilizados y analizados en este estudio son propiedad del Laboratorio de Fertilidad de suelos de la Universidad Central de Venezuela y están disponibles del autor correspondiente a solicitud razonable.

\section{CONFLICTO DE INTERESES}

Los autores declaran que no tienen intereses en competencia.

\section{FONDOS}

Se contó con el financiamiento y apoyo de los Laboratorios de Fertilidad de suelos y Agrología de la Facultad de Agronomía de la Universidad Central de Venezuela, Núcleo Maracay.

\section{CONTRIBUCIÓN DE LOS AUTORES}

Conceptualización: Magaly Ruiz-Dager (M.R.D). Análisis: M.R.D. y Jorge Paolini (J.P). Metodología: M.R.D. y J.P. Escritura y preparación del borrador original: M.R.D. y J.P. ambos autores han leído y están de acuerdo con la versión final publicada de este manuscrito.

\section{AGRADECIMIENTOS}

Magaly Ruiz-Dager agradece a las autoridades de la Universidad Nacional Experimental Rómulo Gallegos por el apoyo administrativo y al Postgrado de Ciencia del Suelo de la Universidad Central de Venezuela por la formación académica adquirida durante el desarrollo de este trabajo. Al Dr. Roberto Villafañe y al profesor Franky Méndez por su contribución en la selección y el muestreo de los suelos estudiados, y por la información relativa a los mismos, así como a los propietarios de las fincas muestreadas por el acceso a sus tierras y el apoyo logístico en el campo. A la TSU Ana Torres y la ingeniera Linda Marilyn Aular, por su ayuda en la realización de los análisis de laboratorio. Los autores agradecen al editor y los revisores de Terra Latinoamericana por las observaciones para mejorar este artículo.

\section{LITERATURA CITADA}

Acuña, O., Peña, W., Serrano, E., Pocasangre, L., Rosales, F. E., Delgado, E. J., Trejos, J., \& Segura, A. (2006). La importancia de los microorganismos en la calidad y salud del suelo. In Proceedings XVII Reunião Internacional da Associação para a Cooperação nas Pesquisas sobre Banana no Caribe e ná América Tropical (ACORBAT) (pp. 223233). Joinville, Santa Catarina, Brasil.

Alguacil, M. del M., Lozano, Z., Campoy, M. J., \& Roldán, A. (2010). Phosphorus fertilisation management modifies the biodiversity of AM fungi in a tropical savanna forage system. Soil Biology and Biochemistry, 42(7), 1114-1122. https://doi.org/10.1016/j.soilbio.2010.03.012

Anderson, T. H. (2003). Microbial eco-physiological indicators to asses soil quality. Agriculture, Ecosystems and Environment, 98(1-3), 285-293. https://doi.org/10.1016/ S0167-8809(03)00088-4

Anderson, T. H., \& Domsch, K. H. (1993). The metabolic quotient for $\mathrm{CO}_{2}\left(\mathrm{qCO}_{2}\right)$ as a specific activity parameter to assess the effects of environmental conditions, such as $\mathrm{pH}$, on the microbial biomass of forest soils. Soil Biology and Biochemistry, 25(3), 393-395. https://doi.org/10.1016/00380717(93)90140-7

Anderson, T. H., \& Domsch, K. H. (2010). Soil microbial biomass: the eco-physiological approach. Soil Biology and Biochemistry, 42(12), 2039-2043. https://doi.org/10.1016/j. soilbio.2010.06.026

Astier-Calderón, M., Maass-Moreno, M., \& Etchevers-Barra, J. (2002). Derivación de indicadores de calidad de suelos en el contexto de la agricultura sustentable. Agrociencia, 36(5), 605-620.

Bini, D., Alcantara dos Santos, C., Banhos do Carmo, K., Kishino, N., Andrade, G., Zangaro, W., \& Nogueira, M. A. (2013). Effects of land use on soil organic carbon and microbial processes associated with soil health in southern Brazil. European Journal of Soil Biology, 55, 117-123. https://doi.org/10.1016/j.ejsobi.2012.12.010 
Borges, L. de A. B., Ramos, M. L. G., Vivaldi, L. J., Fernandes, P. M., Madari, B. E., Soares, R. A. B., \& Fontoura, P. R. (2014). Impact of sugarcane cultivation on the biological attributes of an oxisol in the brazilian savannah. Bioscience Journal, 30(5), 1459-1473.

Cardoso, E. J. B. N., Vasconcellos, R. L. F., Bini, D., Miyauchi, M. Y. H., dos Santos, C. A., Alves, P. R. L., ... Nogueira, M. A. (2013). Soil health: looking for sustainable indicators. What should be considered to assess the effects of use and management on soil health? Scientia Agricola, 70(4), 274289. https://doi.org/10.1590/S0103-90162013000400009

Casanova-Olivo, E. F. (2005). Introducción a la ciencia del suelo. Caracas, Venezuela: CDCH-UCV. ISBN: 9789800023143

Cherubin, M. R., Karlen, D. L., Franco, A. L. C., Cerri, C. E. P., Tormena, C. A., \& Cerri, C. C. (2016). Soil management assessment framework (SMAF) evaluation of brazilian sugarcane expansion on soil quality. Soil Science Society of America Journal, 80(1), 215-226. https://doi.org/10.2136/ sssaj2015.09.0328

Da Silva, D. K. A., Freitas, N. de O., de Souza, R. G., da Silva, F. S. B., Araújo, A. S. F., \& Maia, L. C. (2012). Soil microbial biomass and activity under natural and regenerated forests and conventional sugarcane plantations in Brazil. Geoderma, 189-190, 257-261. https://doi.org/10.1016/j. geoderma.2012.06.014

Dilly, O. (2005). Microbial energetics in soils. In F. Buscot, \& A. Varma (Eds.). Microorganisms in soils: roles in genesis and functions (pp. 123-138). Soil Biology, vol 3. Berlin, FRG: Springer Verlag. https://doi.org/10.1007/3-540-26609-7_6

Dilly, O., Winter, K., Lang, A., \& Munch, J. C. (2001). Energetic eco-physiology of the soil microbiota in two landscapes of southern and northern Germany. Journal of Plant Nutrition and Soil Science, 164(4), 407-413. https:// doi.org/10.1002/1522-2624(200108)164:4<407::AIDJPLN407>3.0.CO;2-9

Dominy, C., Haynes, R., \& van Antwerpen, R. (2002). Loss of soil organic matter and related soil properties under longterm sugarcane production on two contrasting soils. Biology and Fertility of Soils, 36, 350-356. https://doi.org/10.1007/ s00374-002-0538-5

Doran, J. W., \& Safley, M. (1997). Defining and assessing soil health and sustainable productivity. In C. E. Pankhurst, B. M. Doube, \& V. V. S. R. Gupta (Eds.). Biological indicators of soil health (pp. 1-28). Wallingford, UK: CAB International.

Evangelista C. R., Partelli, F. L., Ferreira, E. P. B., \& Pires, F. R. (2013). Atributos microbiológicos do solo na cultura da cana-de-açúcarsob manejo orgânico e convencional Semina: Ciências Agrárias, 34(4), 1549-1562. https://doi. org/10.5433/1679-0359.2013v34n4p1549

Elizalde, G., Viloria, J., \& Rosales, A. (2007). Geografía de suelos de Venezuela. Geovenezuela, 2, 402-537.

FAO (Ed.). (2017). Carbono orgánico del suelo: el potencial oculto. Roma, Italia: Organización de las Naciones Unidas para la Alimentación y Agricultura. ISBN: 978-92-5-3096817

FAO (Ed.). (2018). Guía de buenas prácticas para la gestión y uso sostenible de los suelos en áreas rurales. Bogotá, Colombia: Organización de las Naciones Unidas para la Alimentación y la Agricultura. ISBN: 978-92-5-130425-9
Fialho, J. S., Gomes, V. F. F., de Oliveira, T. S., \& da Silva Júnior, J. M. T. (2006). Indicadores da qualidade do solo em áreas sobvegetação natural e cultivo de bananeiras na Chapada do Apodi-CE. Revista Ciência Agronômica, 37(3), 250-257.

Francaviglia, R., Renzi, G., Ledda, L., \& Benedetti, A. (2017). Organic carbon pools and soil biological fertility are affected by land use intensity in Mediterranean ecosystems of Sardinia, Italy. Science of the Total Environment, 599-600, 789-796. https://doi.org/10.1016/j.scitotenv.2017.05.021

Franzluebbers, A. J. (2004). Tillage and residue management effects on soil organic matter. In F. Magdoff, \& R. R. Weil (Eds.). Soil organic matter in sustainable agriculture (pp. 227-268). Boca Raton, FL: CRC Press.

García-Oliva, F., Gallardo Lancho, J. F., Montaño, N. M., \& Islas, P. (2006). Soil carbon and nitrogen dynamics followed by a forest-to-pasture conversion in western Mexico. Agroforestry Systems, 66(2), 93-100. https://doi.org/10.1007/s10457-0052917-z

Goyal, S., Mishra, M. M., Dhankar, S. S., Kapoor, K. K., \& Batra, R. 1993. Microbial biomass turnover and enzyme activities following the application of farmyard manure to field soils with and without previous long-term applications. Biology and Fertility of Soils, 15(1), 60-64. https://doi.org/10.1007/ bf00336290

González-García, H., González-Pedraza, A. F., Atencio, J., \& Soto, A. (2021). Evaluación de calidad de suelos plataneros a través de la actividad microbiana en el sur del lago de Maracaibo, estado de Zulia, Venezuela. Revista De La Facultad De Agronomía De La Universidad Del Zulia, 38(2), 216-240. https://doi.org/10.47280/RevFacAgron(LUZ).v38.n.2.01

Guevara-Pérez, E., Guevara-Bello, J. E., \& García, E. (2008). Régimen climático y patrón espacial de las lluvias en la cuenca del Lago de Valencia, Venezuela. Revista de Climatología, 8, 29-40.

Heanes, D. L. (1984). Determination of total organic-C in soils by an improved chromic acid digestion and spectrophotometric procedure. Communications in Soil Science and Plant Analysis, 15(10), 1191-1213. https://doi. org/10.1080/00103628409367551

Hernández-Hernández, R. M., Pulido-Moncada, M., Caballero, R., Cabriales, E., Castro, I., Ramírez, E., ... Mendoza, B. (2013). Influencia del cambio de uso de la tierra sobre las sustancias húmicas y la estabilidad de los agregados en suelos de sabanas y bosques tropicales. Revista De La Facultad De Agronomía De La Universidad Del Zulia, 30(4), 551-572.

Mazzarino, M. J., Szott, L., \& Jimenez, M. (1993). Dynamics of soil total $\mathrm{C}$ and $\mathrm{N}$, microbial biomass, and water-soluble $\mathrm{C}$ in tropical agroecosystems. Soil Biology and Biochemistry, 25(2), 205-214. https://doi.org/10.1016/0038-0717(93)90028-a

Nannipieri, P., Greco, S., \& Ceccanti, B. (1990). Ecological significance of the biological activity in soil. In J. M. Bollag, \& G. Stotzky (Eds.). Soil biochemistry (pp. 293-355) volume 6. New York, NY: Marcel Dekker

NRC (National Research Council). (2010). Toward Sustainable Agricultural Systems in the 21 st Century. Washington, DC: The National Academies Press. https://doi.org/10.17226/12832

Novak, E., Carvalho, L. A., Santiago, E. F., \& Portilho, I. I. R. (2017). Chemical and microbiological attributes under different soil cover. CERNE, 23(1), 19-30. https://doi.org/10. $1590 / 01047760201723012228$ 
Paredes Junior, F. P., Portilho, I. I. R., \& Mercante, F. M. (2015). Atributos microbiológicos de um latossolo sob cultivo de canade-açúcar com e sem queima da palhada. Semina: Ciências Agrárias, 36(1), 151-164. https://doi.org/10.5433/1679$0359.2015 \mathrm{v} 36 \mathrm{n} 1 \mathrm{p} 151$

Paolini-Gómez, J. E. (2018). Actividad microbiológica y biomasa microbiana en suelos cafetaleros de los Andes venezolanos. Terra Latinoamericana, 36(1), 13-22. https:// doi.org/10.28940/terra.v36i1.257

Pôrto, M. L., Alves, J. do C., Diniz, A. A., de Souza, A. P., \& Santos, D. (2009). Indicadores biológicos de qualidade do solo em diferentes sistemas de uso no brejoparaibano. Ciência e Agrotecnologia, Lavras, 33(4), 1011-1017. https://doi. org/10.1590/S1413-70542009000400010

Rietz, D. N., \& Haynes, R. J. (2003). Effects of irrigation-induced salinity and sodicity on soil microbial activity. Soil Biology and Biochemistry, 35(6), 845-854. https://doi.org/10.1016/ s0038-0717(03)00125-1

Rivera-Cruz, M. C., Trujillo-García, A., Córdova-Ballona, G., Kohler, J., Caravaca, F., \& Roldán, A. (2008). Poultry manure and banana waste are effective biofertilizer carriers for promoting plant growth and soil sustainability in banana crops. Soil Biology and Biochemistry, 40(12), 3092-3095. https://doi.org/10.1016/j.soilbio.2008.09.003

Ruiz, M., \& Paolini, J. (2004). Efecto del cultivo y el agua de riego sobre el carbono de la biomasa microbiana. Agronomía Tropical (Maracay), 54(2), 161-178.

Sant'Anna, S. A. C., Fernandes, M. F., Ivo, W. M. P. M., \& Costa, J. L. S. (2009). Evaluation of soil quality indicators in sugarcane management in sandy loam soil. Pedosphere, 19(3), 312-322. https://doi.org/10.1016/s1002-0160(09)60122-3
Saviozzi, A., Levi-Minzi, R., Cardelli, R., \& Riffaldi, R. (2001). A comparison of soil quality in adjacent cultivated, forest and native grassland soils. Plant and Soil, 233(2), 251-259. https://doi.org/10.1023/a:1010526209076

Silva, R. R. da, Silva, M. L. N., Cardoso, E. L., Moreira, F. M. de S., Curi, N., \& Alovisi, A. M. T. (2010). Biomassa e atividade microbiana em solo sob diferentes sistemas de manejo na região fisiográfica Campos das Vertentes - MG. Revista Brasileira de Ciencia do Solo, 34(5), 1585-1592. https://doi. org/10.1590/s0100-06832010000500011

Souza, R. A., Telles, T. S., Machado, W., Hungria, M., Filho, J. T., \& Guimaraes, M. F. (2012). Effects of sugarcane harvesting with burning on the chemical and microbiological properties of the soil. Agriculture, Ecosystems \& Environment, 155(1), 1-6. https://doi.org/10.1016/j.agee.2012.03.012

Stirling, G. R., Moody, P. W., \& Stirling, A. M. (2010). The impact of an improved sugarcane farming system on chemical, biochemical and biological properties associated with soil health. Applied Soil Ecology, 46(3), 470-477. https://doi. org/10.1016/j.apsoil.2010.08.015

Wardle, D. A., \& Ghani, A. (1995). A critique of the microbial quotient $\left(\mathrm{qCO}_{2}\right)$ as a bioindicator of disturbance and ecosystem development. Soil Biology and Biochemistry, 27(12), 16011610. https://doi.org/10.1016/0038-0717(95)00093-t

Zhong, S., Zeng, H., \& Jin, Z. (2015). Soil microbiological and biochemical properties as affected by different long-term banana-based rotations in the tropics. Pedosphere, 25(6), 868877. https://doi.org/10.1016/s1002-0160(15)30067-9 